\title{
How social contexts affect cognition: mentalizing interferes with sense of agency during voluntary action
}

Nura Sidarus ${ }^{1}$, Patrick Haggard ${ }^{2}$, Frederike Beyer ${ }^{3 *}$

${ }^{1}$ Institut Jean Nicod, Département d'Études Cognitives, Ecole Normale Supérieure, EHESS, CNRS, PSL University

${ }^{2}$ Institute of Cognitive Neuroscience, University College London

${ }^{3}$ Department of Biological and Experimental Psychology, Queen Mary University of London

* Corresponding author

Dr. Frederike Beyer

Department of Biological and Experimental Psychology

School of Biological and Chemical Sciences

Queen Mary University of London

Mile End Road

E1 4NS, London

Phone: +44 (0)20 78827183

Email: f.beyer@qmul.ac.uk

Word count: 4,854 


\begin{abstract}
Living in complex social structures, humans have evolved a unique aptitude for mentalizing. On one view, mentalizing has shaped neurocognitive evolution, yet, little is known about how mentalizing interacts with other cognitive processes. For social animals, actions of one individual often impact others. "Sense of agency" refers to the feeling of control over the outcomes of one's actions, providing a precursor of responsibility. Here, we test a model of how social context influences this key feature of human action, even when action outcomes are not specifically social.

We show that the presence of another potential agent reduces sense of agency for positive and negative outcomes. This dissociates social modulation of sense of agency from classical selfserving bias, since the latter would reduce sense of agency only for undesirable outcomes.

Instead, we propose that the cognitive load involved in decision-making is increased by the requirement to mentalize, and compute the possible actions of others. In a second experiment, we test this hypothesis by comparing two situations, in which participants either need to consider potential actions of another person, or potential failures of a causal mechanism. We find reduced sense of agency only in the social condition, suggesting that the presence of another intentional agent has a unique influence on the cognitive processes underlying one's own voluntary action. Previous work primarily focussed on social facilitation of human cognition. However, when people must incorporate potential actions of others into their decision-making, we show that the resulting cognitive load reduces individuals' feelings of control.
\end{abstract}




\section{Introduction}

Compared to non-human primates, humans live in highly complex cooperative social structures, a fact that is linked to the development of sophisticated mentalizing skills during recent evolution (Hare, 2011). The evolution of the human brain appears directly driven by the need for complex social cognition, with a wide-ranging network of neural structures (medial prefrontal cortex; temporo-parietal junction; temporal poles; precuneus) supporting mentalizing processes (Schurz, Radua, Aichhorn, Richlan, \& Perner, 2014). This would suggest that the mentalizing processes underlying social interaction have shaped other, non-social cognitive processes. In that case, consistent and characteristic interactions between mentalizing and non-social cognition should exists. However, the tasks used in much previous research on this topic often assumed this interaction, rather than directly test it - often requiring social cognition as an explicit element of the task. For example, when participants need to learn to predict another agent's behaviour, mentalizing is indeed related to better performance (Devaine, Hollard, \& Daunizeau, 2014). Here, we investigate a framework of how social settings may influence human action processing. A central aspect of action control, which becomes particularly important in social settings, is the "sense of agency", or the feeling that we are in control of our actions and their outcomes. Sense of agency is an essential feature of normal human behaviour, and has wide structuring effects on cognitive processes, from perception (Tsakiris \& Haggard, 2005) to outcome evaluation (Bednark \& Franz, 2014). Further, sense of agency plays a particularly important role in social settings, since it is essential for attribution of responsibility (Frith \& Haggard, 2018). In previous studies, we have shown that the mere presence of another potential agent reduces sense of agency and outcome monitoring (Beyer, Sidarus, Bonicalzi, \& Haggard, 2017), confirming the hypothesis of strong interactions between mentalizing and wider cognition. Interestingly, this effect was associated with increased activation of the precuneus (Beyer, 
Sidarus, Fleming, \& Haggard, 2018), a key node in the mentalizing network. Therefore, we have argued that, in social contexts, mentalizing interferes with decision-making processes, as the potential actions of other agents must also be considered, thereby reducing sense of agency. This hypothesis draws on previous work showing that sense of agency is reduced by dysfluency in action selection (Sidarus, Chambon, \& Haggard, 2013; Sidarus, Vuorre, \& Haggard, 2017; Sidarus \& Haggard, 2016) and increased cognitive load (Hon, Poh, \& Soon, 2013; Howard, Edwards, \& Bayliss, 2016; Wen, Yamashita, \& Asama, 2016).

However, our previous studies on the effect of social context on sense of agency only involved negative action outcomes. Thus, reduced sense of agency could reflect a self-serving bias (Bandura, 2002): when another agent is present, this offers the opportunity to attribute negative outcomes away from the self. Moreover, previous studies lacked a non-social control, so that the only influence on participants' decisions was a social agent. This means that social modulation of sense of agency cannot be distinguished from an increase in the general complexity of decisionmaking.

In two experiments, we test how mentalizing influences the human sense of agency. First, we test whether the presence of another agent reduces sense of agency similarly for positive and negative action outcomes. In a second experiment, we create two experimental setups that are identical in terms of the events that participants experience, but differ in their instructions. Namely, one group of participants receive instructions that any external influence on the task is caused by another person. The other group is instructed that any influence is caused by a faulty mechanical device. This allows for a direct test of the influence of social cognition on sense of agency. We discuss the implications of our findings for common practices of education and for our understanding of social development. 


\section{Experiment 1}

The first experiment served to test the prediction, from our model of mentalizing influences on sense of agency, that social context should similarly reduce sense of agency for positive and negative action outcomes. One group of participants performed a "gain" version of the task (fig. 1), another group performed a "loss" version. In each trial, participants had to make an action in order to stop an inflating balloon from bursting. In the 'gain' frame, each action resulted in a monetary gain. In the 'loss' frame, each action was associated with a monetary cost. In social context trials, another putative player (represented by an avatar on the screen) could act instead of the participant, which always resulted in the best outcome for the participant (fig. 1).

\section{$\underline{\text { Methods }}$}

\section{Participants \& procedure}

Our previous studies (Beyer et al., 2017, 2018) showed large effect sizes for the within subject effect of social context on agency ratings. Here, we were focused on the effects of a between group manipulation (gain/loss frame), and estimated the sample size required for achieving $80 \%$ power for detecting a large effect size of $d=.8(\alpha=.05$; recommended sample size 21 subjects per group). 44 healthy volunteers were recruited for the experiment. 22 participants performed the task in the gain frame, 22 performed the task in the loss frame. One participant in the gain frame was excluded from the analysis due to low trial numbers. Thus, data of 43 participants were included in the analysis.

Participants were invited into the lab in pairs, received instructions together and were told that they would be playing together in the experiment. They were then brought into separate computer cubicles to perform the task. After the task, participants filled out a post-experimental 
questionnaire, were fully debriefed and paid $£ 7.50$ for their participation, plus a bonus based on their task performance.

All participants gave written informed consent and the study was approved by the local ethics committee.

Task

The task (figure 1) was adapted from that used in (Beyer et al., 2017) and modelled after the balloon analogue risk task (Lejuez et al., 2002). In each trial, participants saw a small balloon in the centre of the computer screen, which inflated at constant speed. At a randomly determined size that varied from trial to trial, the balloon popped. At any time, the participant could press the left button on a standard computer mouse to stop the balloon.

To the left of the balloon, the participant saw an avatar representing themselves. To the right of the balloon, the participant saw either a coloured rectangle (in non-social trials), or another avatar representing their alleged co-player (in social trials). In social trials, the co-player could sometimes stop the balloon before, and thus instead of, the participant. In each trial, the avatar belonging to the player who stopped the balloon was marked by a red rectangle as soon as a response was made.

In the loss frame, the payoff structure was as follows: if the balloon burst, the participant lost 20 points (and was told that in social trials, so would their co-player); if the participant stopped the balloon, they lost 1-20 points depending on the size of the balloon (the bigger the balloon, the fewer points they lost); in social trials, if the co-player stopped the balloon, the participant lost 0 points. In the gain frame, the payoff was as follows: if the balloon burst, the participant earned 0 points; if the participant stopped the balloon, they earned 1-20 points (the bigger the balloon, the more points they earned); in social trials, if the co-player stopped the balloon, the participant earned 20 points. 
Thus, in both frames, the best outcome was obtained by the co-player's action, the worst if neither player acted, and an outcome in-between these extremes if the participant acted, depending on balloon size.

At the end of each trial, participants rated how much control they felt they had over the outcome of that trial, on a visual analogue scale ranging from 'no control' to 'complete control'. Participants were instructed that the outcome referred to the number of points they lost on that trial, rather than whether the balloon popped or not.

The co-player's behaviour was pre-programmed, such that they would only stop the balloon if the participant had stopped the balloon on the majority of social trials of that block (i.e. if the participant had stopped the balloon on at least one social trial more, than the co-player). If this was the case, the co-player stopped the balloon with a likelihood of about $66 \%$.

Participants played 4 blocks of 30 trials each. In each block, 15 social and 15 non-social trials were intermixed randomly, resulting in 60 trials per experimental condition. 


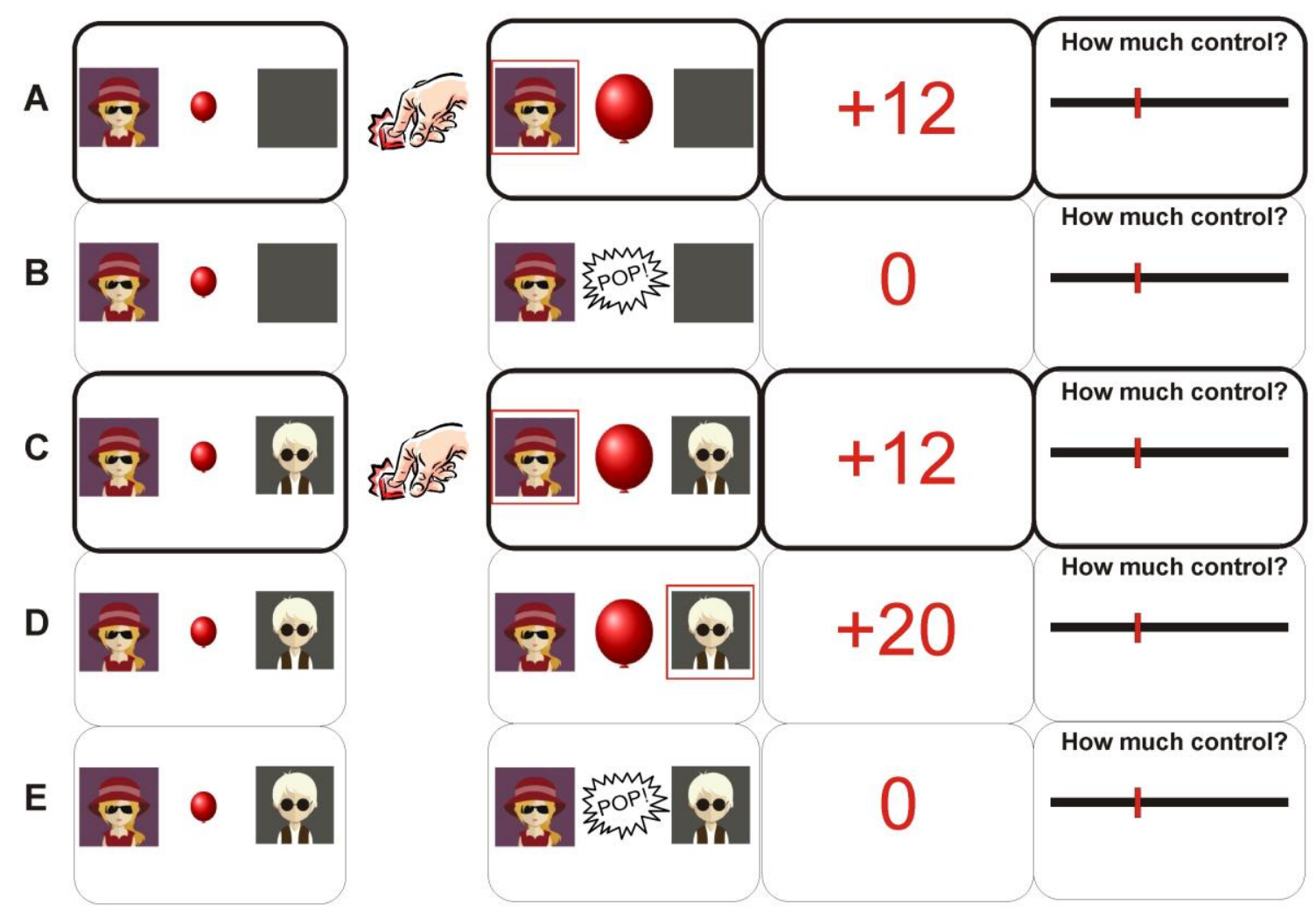

Figure 1: task outline for experiment 1. Figure shows the different conditions for the task in the gain frame. Co-player absent context: participant successfully stops the balloon and earns the respective number of points (A); balloon pops, participant earns 0 points (B). Co-player present condition: participant successfully stops the balloon and earns the respective number of points (C); co-player stops the balloon, participant earns the maximum number of points (D); balloon pops, participant earns 0 points (E). Analyses focused on trial types $\mathbf{A}$ and $\mathbf{C}$. Task structure was identical for the loss frame, except for outcome value (which ranged from 0 to -20). In both gain and loss frames, participants obtain the best outcome when the co-player acts, and the worst outcome when the balloon bursts.

\section{Data analysis}

Our analysis focused on agency ratings in trials in which the participant successfully stopped the balloon before it burst, as these trials are comparable between contexts in which the co-player was present or absent. We used mixed linear models in R (R Development Core Team, 2008), using R Studio (https://www.rstudio.com/). 
We entered trial-wise agency ratings as the dependent variable, modelled by gain and loss frame as between-subject factor (coded as -.5 for loss frame and .5 as gain frame), and outcome magnitude (z-standardized within participants) and presence of co-player (coded as .5 for absent and -.5 for present) as within-subject factors.

\section{$\underline{\text { Results }}$}

General task performance did not differ between groups (see supplementary material SM1a). Our analyses focused on trials in which the participant stopped the balloon. For these trials, event sequences and action-outcome contingencies were identical for social and non-social contexts. We set up a mixed linear model analysis of sense of agency ratings, with context (social vs. nonsocial) and outcome magnitude (z-standardized within participants) as within-subject factors and frame group ('gain' vs. 'loss') as a between-subject factor.

We found a significant main effect of outcome $\left(b=5.3, C I=3.9 / 6.8, t_{41.3}=7.1, p<.001\right)$, a main effect of context (co-player absent or present; $\mathrm{b}=3.0, \mathrm{CI}=1.2 / 4.8, \mathrm{t}_{41.1}=3.3, \mathrm{p}=.002$ ), and an outcome ${ }^{*}$ context interaction $\left(\mathrm{b}=1.8, \mathrm{CI}=0.7 / 2.9, \mathrm{t}_{39.2}=3.1, \mathrm{p}=.003\right.$; figure 2$) . \mathrm{We}$ found no main effect of gain/loss group $(\mathrm{p}=.284)$, and no significant interaction involving the group factor (all p > .29; see supplementary table SM1b for full test statistics).

In accordance with previous findings (Beyer et al., 2017, 2018), participants felt more in control over better outcomes, and felt less in control in the social context compared to the non-social one. Importantly, the interaction between outcome magnitude and social context demonstrates that a self-serving bias, leading to a strategic displacement of agency for undesirable outcomes, cannot explain the reduction in agency ratings in the social context. 

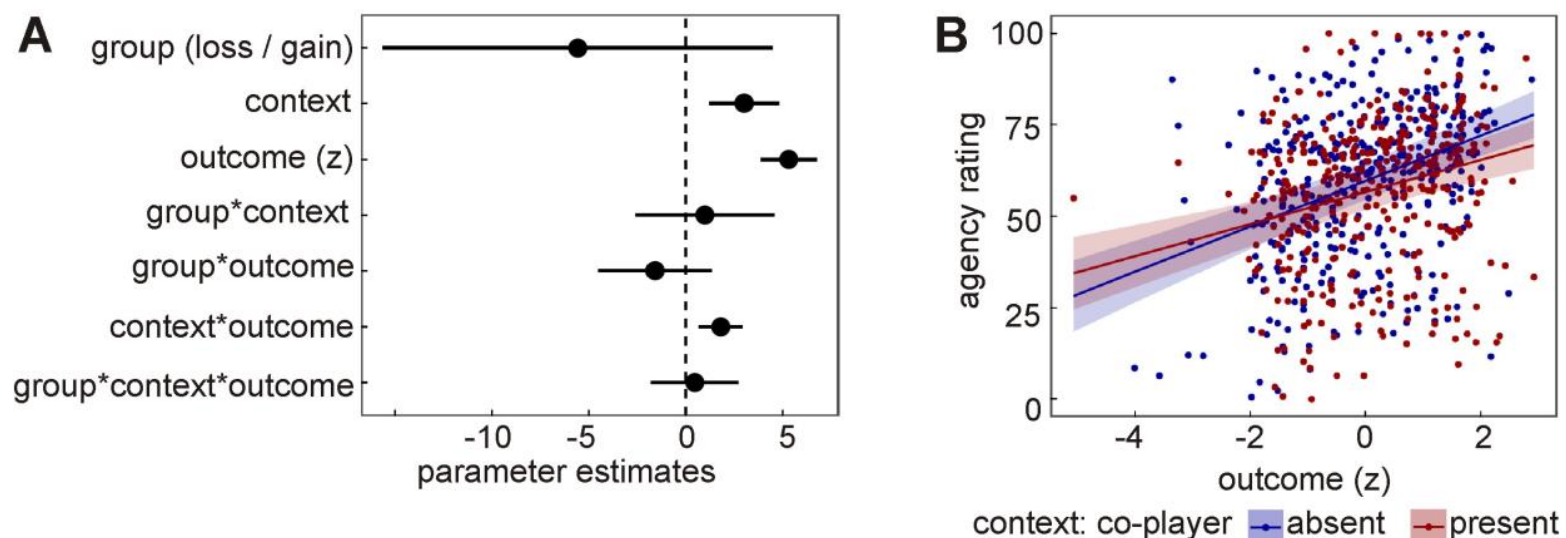

Figure 2: results for experiment 1. A. Parameter estimates for the mixed model analysis. The 'Context' factor refers to the presence or absence of the co-player in a given trial. B. The context*outcome magnitude interaction effect on agency ratings, collapsed across loss and gain frame groups.

As figure $2 \mathrm{~B}$ shows, the difference in agency ratings between social and non-social context increased for better outcomes, and was absent for particularly bad outcomes. To test the interaction effect, we performed one-sample t-tests on point estimates for the beta values of social context effects (Tom, Bosker, \& Bosker, 1999), for average outcomes, and also outcomes one standard deviation above and below the average. This confirmed that there was a significant effect of context for good outcomes $\left(+1\right.$ standard deviations $(\mathrm{SD})$ above mean; $\left.\mathrm{t}_{42}=4.5, \mathrm{p}<.001\right)$ and for average outcomes $\left(\mathrm{t}_{42}=3.1 ; \mathrm{p}=.002\right)$, but not for bad outcomes $\left(-1\right.$ SD below mean; $\mathrm{t}_{42}=$ $1.1, \mathrm{p}=.270)$.

Our findings are in line with the hypothesis that the reduction in sense of agency in social contexts is driven by mentalizing processes, rather than self-serving bias, and should thus be similar for actions with positive and negative outcomes. Across gain and loss frame settings, participants felt less in control over the consequences of their own actions when another potential agent was present. 


\section{Experiment 2}

If people feel less in control in social action contexts because mentalizing processes interfere with decision-making, then this effect should be specific for social influences. However, if mere uncertainty prior to the action or post-hoc counterfactual thinking leads to the subjective loss of agency, then this should also be observed for non-social sources of alternative trial outcomes. We compared the agency-reducing effect of the presence of an alternative agent between two task settings (figure 3). One was a social setting similar to the one described for experiment 1 ; the other setting was identical in all aspects, except that the alternative agent was introduced as nonintentional and non-social.

In the social version, the presence or absence of the alternative agent was marked by an avatar next to the balloon, as described for experiment 1. In the non-social version, participants saw the image of an air pump next to either side of the balloon. That pump was coloured either green or blue. Participants were instructed that the green pump was new, and the blue pump was old. The green pump would always inflate the balloon until it popped, unless the participant acted. The blue pump might, on some trials, break down before the balloon was fully inflated, in which case the participant would not lose any points.

The social co-player and the non-social pump were programmed in the same way: the alternative agent would only act if the participant had acted on the majority of social trials and for a maximum of 3 trials per block. The only difference between task versions was that the pump was introduced as a non-social agent, thus not encouraging the engagement of mentalizing processes. 


\section{$\underline{\text { Methods }}$}

\section{Participants \& procedure}

48 healthy volunteers were recruited for the experiment. 24 participants performed the task in the social condition, 24 performed the task in the non-social. For the social version, participants were invited into the lab in pairs, received instructions together and were told that they would be playing together in the experiment. They were then brought into separate computer cubicles to perform the task. For the non-social version, participants were recruited alone or in pairs, but were not told they would be playing together. After the task, participants filled out a postexperimental questionnaire, were fully debriefed and paid $£ 7.50$ per hour for their participation, plus a bonus based on their task performance.

All participants gave written informed consent and the study was approved by the local ethics committee.

\section{Task}

The task (figure 3) was similar to that of experiment 1, with a few alterations making it more similar to that used in (Beyer et al., 2018). Mainly, an image of a pin was presented above the balloon, such that the balloon would always pop at the same size, when it touched the pin. The balloon would inflate at variable speed and speed up unpredictably at some point of a given trial, in order to make it risky to wait until the maximum size possible. The payoff structure was that of a loss frame: if the balloon popped, participants lost 80-99 points; if they stopped the balloon, they lost 1-60 points; in trials with the alternative agent, if that agent stopped the balloon, participants lost 0 points. The other agent (co-player / old pump) was programmed to stop the balloon with a likelihood of about $70 \%$, if the participant had acted on the majority of social trials, and for a maximum of 3 trials per block. 
Participants completed three blocks of 20 trials each with 10 agent absent (co-player absent / new pump) and 10 agent present (co-player present / old pump) trials per block, randomized on a trialwise basis.

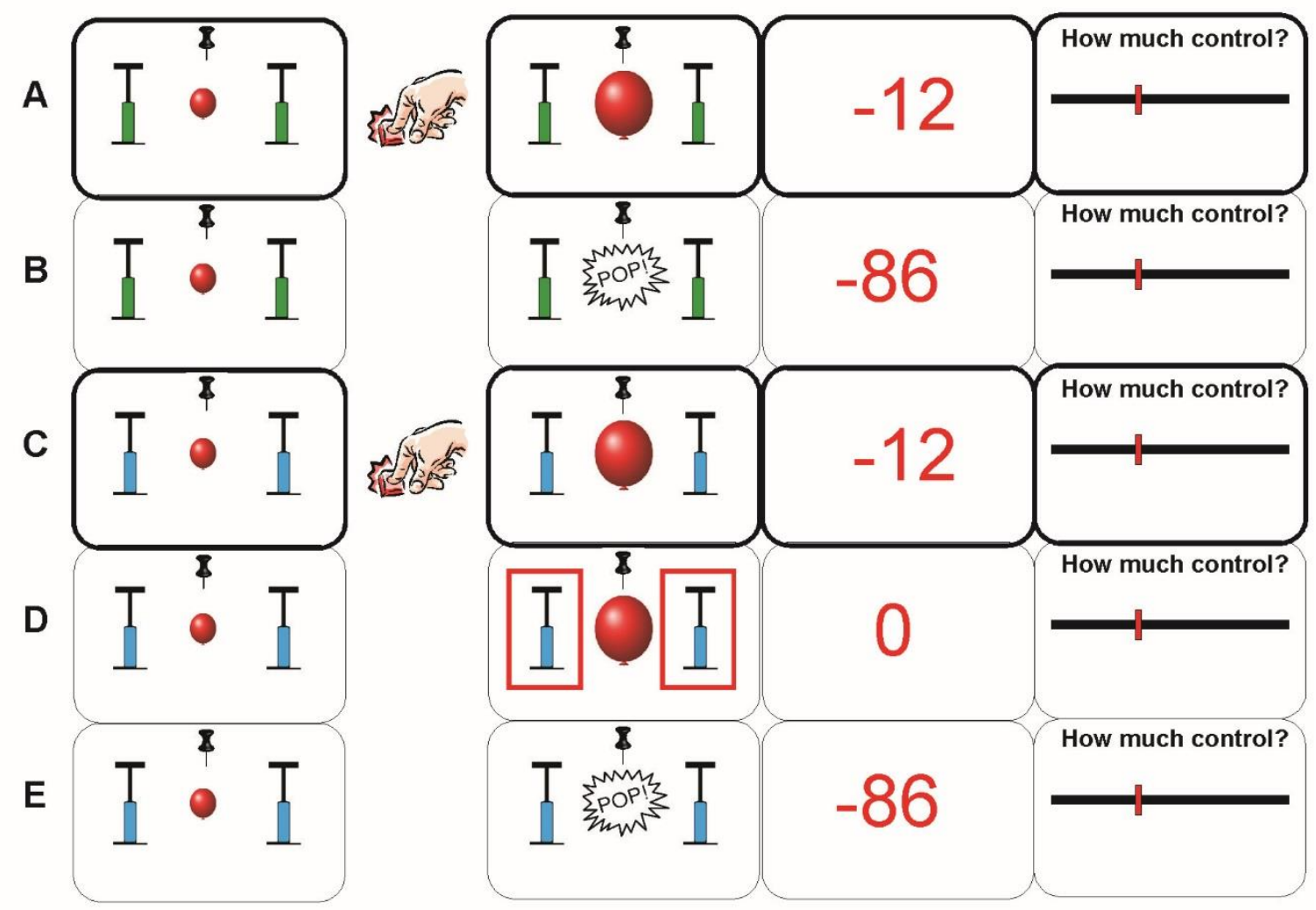

Figure 3: task outline for experiment 2. Figure shows the different conditions for the nonsocial task version. Alternative agent absent context: participant successfully stops the balloon and loses the respective number of points (A); balloon pops, participant loses up to 99 points (B). Alternative agent present condition: participant successfully stops the balloon and loses the respective number of points $(\mathbf{C})$; pump breaks down, participant loses no points (D); balloon pops, participant loses up to 99 points (E). Analyses focused on trial types $\mathbf{A}$ and $\mathbf{C}$. For the social task version, avatars were presented as in experiment 1, as shown in figure 1 (with the addition of a pin above the balloon, as shown here). Outcome magnitude was identical to that in the non-social task version shown here. 


\section{Data analysis}

Analysis was conducted as in experiment 1, with alternative agent (present vs. absent) and outcome magnitude (z-standardized) as within-subject factors, and framing condition (social vs. non-social) as between-subject factor.

\section{$\underline{\text { Results }}$}

Comparing task performance between task versions showed, most importantly, no difference between social (avatar) and non-social (pump) agent groups in the number of trials in which the alternative agent acted $\left(\mathrm{M}=7.6 / 7.5 ; \mathrm{SD}=1.6 / 1.6 ; \mathrm{t}_{46}=0.4, \mathrm{p}=.656\right)$. Thus, participants in the social and non-social versions experienced the same level of external influence and could form similar expectations about the probability of the balloon stopping 'on its own'. Considering the number of trials in which the participant did act, a group by context mixed ANOVA showed significant main effects of group $\left(\mathrm{F}_{1,46}=8.0 ; \mathrm{p}=.007, \eta_{\mathrm{p}}^{2}=.15\right)$, context $\left(\mathrm{F}_{1,46}=236.3 ; \mathrm{p}<.001\right.$, $\left.\eta_{\mathrm{p}}{ }^{2}=.84\right)$, and a significant interaction $\left(\mathrm{F}_{1,46}=5.6 ; \mathrm{p}=.023, \eta_{\mathrm{p}}{ }^{2}=.11\right)$. Post-hoc tests revealed that, when the alternative agent was present, participants in the social task frame acted less frequently than participants in the non-social frame $\left(M=16.3 / 19.2 ; \mathrm{SD}=3.3 / 3.0 ; \mathrm{t}_{46}=-3.2 ; \mathrm{p}\right.$ $<.01)$, while there was no difference between groups when the alternative agent was absent $(\mathrm{M}=$ $\left.24.6 / 25.3 ; \mathrm{SD}=2.5 / 1.9 ; \mathrm{t}_{46}=-1.0 ; \mathrm{p}=.304\right)$. While, as is to be expected, both groups acted less often when the balloon could be stopped by the alternative agent (paired t-test for agent present vs. absent, social frame: $\mathrm{t}_{23}=11.7, \mathrm{p}<.001$; non-social frame: $\mathrm{t}_{23}=10.0, \mathrm{p}<.001$ ), this effect was stronger if participants thought they were playing with another person, than if they were playing with a faulty pump. . Thus, even though they had the same experience of external influence on stopping the balloon, participants who believed the alternative agent in that condition to be another person relied more on the other agent to act, relative to participants who 
did not believe that another person was involved. Since both groups had the same number of trials in which the alternative agent acted, acting less often in the agent present condition for the social frame group resulted in a larger number of balloon bursts trials, and hence a slightly inferior task performance, with a lower gain on average (social vs. non-social group: $\mathrm{M}=46.6$ / 70.6; $\left.\mathrm{SD}=33.9 / 21.1 ; \mathrm{t}_{46}=2.9 ; \mathrm{p}=.005\right)$. [See SM2a Task performance for analysis of RTs, which showed no significant effects].

The model of agency ratings (figure 4) with the factors group (social / non-social), context (agent absent / agent present) and outcome (z-standardized) showed a main effect of outcome magnitude $\left(\mathrm{b}=5.13, \mathrm{CI}=3.43 / 6.82, \mathrm{t}_{46.41}=5.93, \mathrm{p}<.001\right)$, as well as a group*context*outcome interaction $\left(b=4.30, \mathrm{CI}=1.19 / 7.42, \mathrm{t}_{84.37}=2.71, \mathrm{p}<.01 ;\right.$ figure 5$)$.

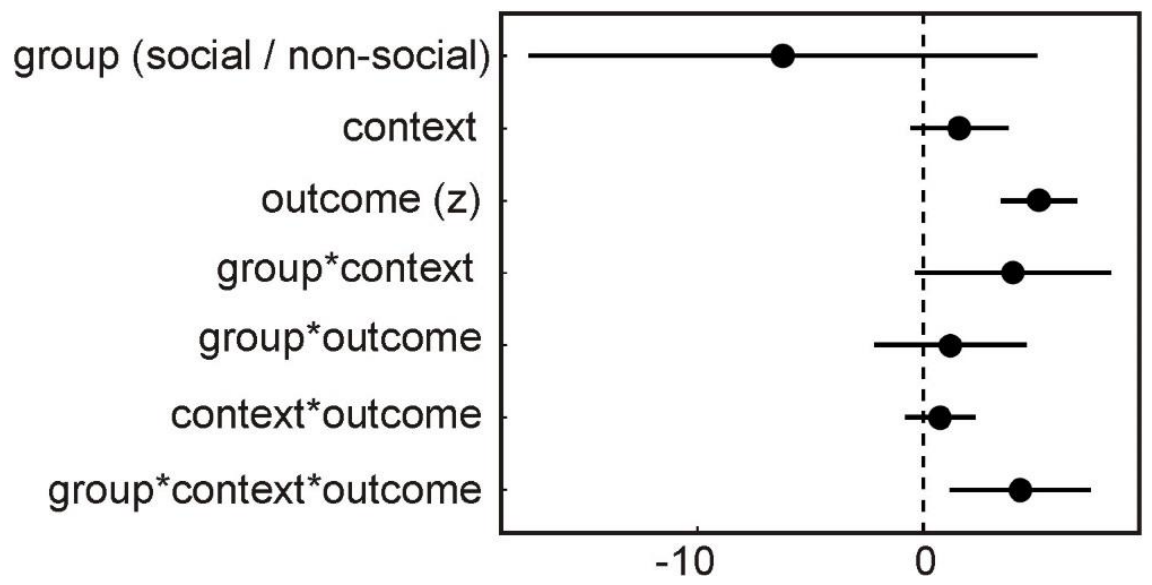

Figure 2: main results for experiment 2. Figure shows the parameter estimates for the mixed effects linear model including the social (avatar) vs. non-social (pump) group factor. 'Context' referse to the presence or absence of the alternative agent (i.e. co-player present/absent, pump old/new).

To further investigate the three-way interaction, we performed separate analyses for the two experimental groups. For the social group, this showed a main effect for outcome $(b=5.75, C I=$ $\left.3.41 / 8.10, \mathrm{t}_{23.4}=4.81, \mathrm{p}<.001\right)$, as well as a significant context ${ }^{*}$ outcome interaction $(\mathrm{b}=2.85$, 
$\left.\mathrm{CI}=0.23 / 5.46, \mathrm{t}_{40.6}=2.14, \mathrm{p}<.05\right)$. As can be seen in figure 5 , this group showed a similar interaction as observed in experiment 1. Agency ratings increased more strongly with better outcomes in the agent-absent context compared to the agent-present context (in which the alleged co-player could have acted), leading to a significant effect of context for good outcomes $\left(\mathrm{t}_{23}=\right.$ $2.28, \mathrm{p}<.05)$, and a marginal effect for average outcomes $\left(\mathrm{t}_{23}=1.77, \mathrm{p}=.091\right)$, but no effect for bad outcomes $\left(\mathrm{t}_{23}=.38, \mathrm{p}=.705\right)$. For the non-social group, there was a significant effect of outcome $\left(\mathrm{b}=4.58, \mathrm{CI}=2.16 / 6.99, \mathrm{t}_{23.1}=3.71, \mathrm{p}=.001\right)$, but no effect of context $(\mathrm{p}=.671)$ and no context*outcome interaction $(\mathrm{p}=.115$; see supplementary tables $\mathrm{SM} 2 \mathrm{~b}, \mathrm{c} \& \mathrm{~d}$ for full test statistics).
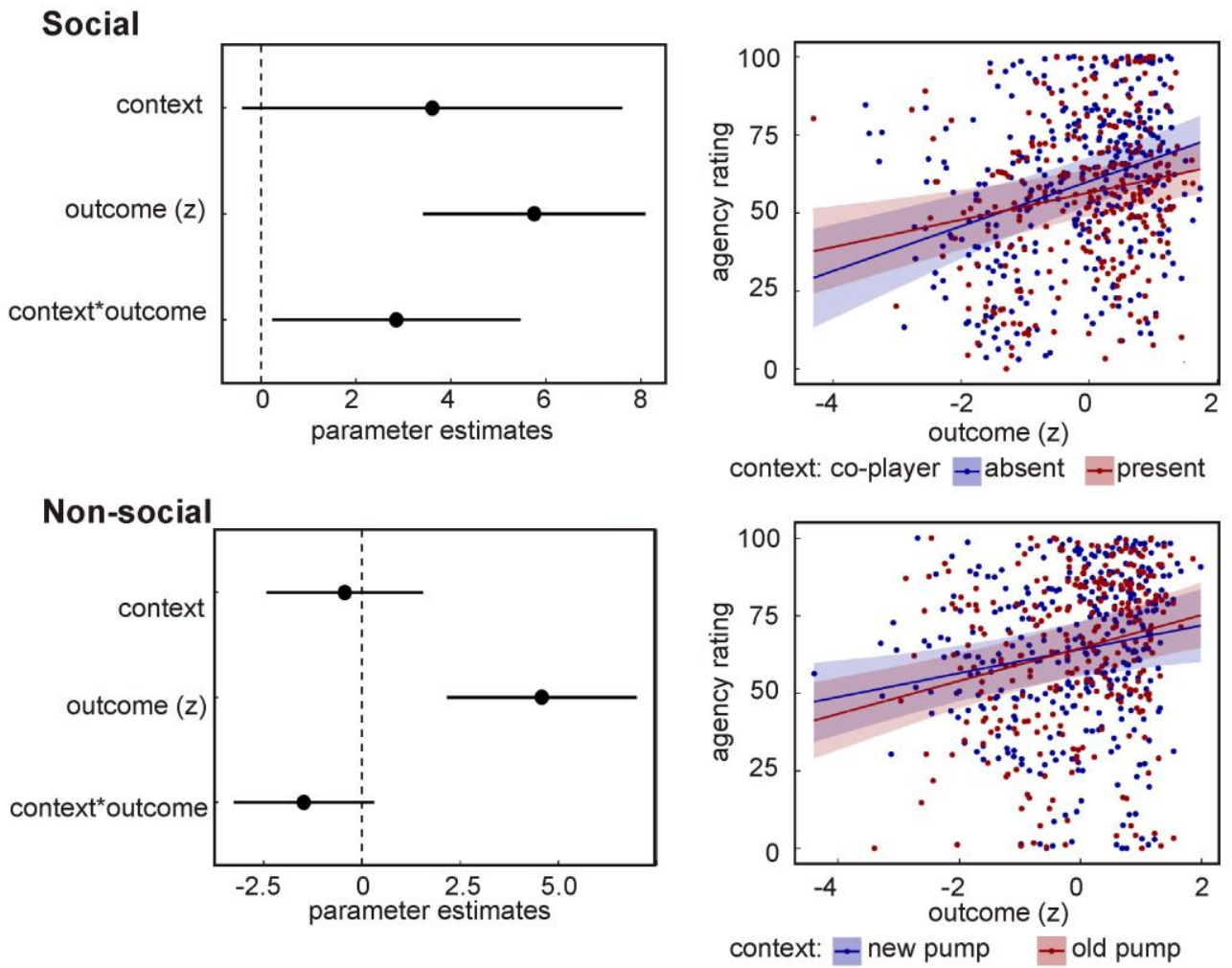

Figure 3: results for separate analysis of social and non-social groups. Figure shows the context (alternative agent present vs. absent) by outcome magnitude interaction effects for the social and non-social experimental groups. 
The results of this experiment show that the reduction in sense of agency due to the presence of another potential agent occurs only when that agent is assumed to be a person (i.e. social agent), and not when it is assumed to be a mere mechanism. When a non-intentional, non-social agent could interfere with the participant's action, no reduction in sense of agency was observed for trials in which the participant successfully acted. Participants also behaved differently towards social agents, relying more on them than on a non-social agent to intervene in response to increasing risk, and to act before the balloon exploded. These findings show that social cognition is indeed a crucial factor in these contextual effects on sense of agency. Alternative explanations for reduced sense of agency in social contexts could be a shift in subjective outcome value (i.e. when a no-loss option is possible, a small negative outcome could be perceived as worse, than when the no-loss option is not available), increased uncertainty of trial outcomes prior to the action, or prior experience of non-control (i.e. the balloon stopping 'on its own') could become associated with the task condition, lowering the overall sense of agency in the presence of the alternative agent. Crucially, these explanations would have predicted the same effect for the nonsocial agent, i.e. the old and faulty pump.

\section{Discussion}

In two experiments, we have investigated the interaction between mentalizing and a key nonsocial aspect of human cognition, namely the emergence of a sense of agency. In a first study, we showed that social context reduces sense of agency, independently of outcome valence. More specifically, in the presence of another potential agent, participants felt less in control over the consequences of their actions, regardless of whether those consequences were positive or negative. In a second study, we showed that this effect relies on the occurrence of mentalizing 
processes, as it was specific for the presence of a social agent. Importantly, in both cases, the alternative agent had no influence on the outcomes of the participant's action.

In the study comparing social and non-social agents, participants experienced the same amount of external influence in the task, that is, the balloon was stopped by the alternative agent in the same number of trials. Yet, the presence of another potential agent only influenced sense of agency when the agent was believed to be a social, intentional entity, compared to a non-living, random one. This shows that a (putative) social presence evokes strong, competing cognitive processes, which are not evoked by alternative non-social sources of action. One plausible explanation for this is that people try to build a model of the other putative social agent's behaviour in order to predict what the other agent will do. Participants would likely mentalize about their co-player's potential behaviour, trying to predict when and why the co-player might act, thus potentially avoiding the cost of acting themselves. The other player's alleged presence may also have increased a priori counterfactual thinking during decision-making ('If I act now, I won't know if the other person would have acted had I waited longer'). In contrast, participants in the non-social condition were less influenced by their previous experience of the faulty pump, and tended to ignore the influence of the pump during decision-making. This may be because participants could not, or did not expect to, form a predictive model about the pump's relevant behaviour. When the potential alternative cause of the balloon stopping was non-social (i.e. the "old pump"), it might seem a priori less predictable, hence, participants might not engage resources in trying to understand its behaviour. Thus, assuming an intentional stance towards the social agent results in continuous efforts at modelling and predicting their behaviour. Attempting to form this additional predictive model disrupts the participant's own decision-making and sense of agency. 
This theory is in line with a range of previous findings showing that increased complexity of a decision-making process, as well as increased working memory demands, decrease the sense of agency (Hon et al., 2013; Howard et al., 2016; Sidarus \& Haggard, 2016; Sidarus et al., 2017; Wen et al., 2016). We propose that the presence of another human agent is a particularly strong source of dysfluency, due to the complexity of cognitive processes induced by their presence. These hypotheses are further supported by the observation that participants relied more on the alternative social agent to act, resulting in more trials in which the balloon popped. This suggests that in addition to deciding when to stop the balloon on a given trial, in the presence of a social agent, participants additionally considered whether they should act at all. This decision would depend on their prediction of the co-player's behaviour. The non-social cause of "action" still increased uncertainty about what might happen in each trial, as the balloon might still stop "on its own". However, participants acted more frequently in this condition, experiencing fewer balloon burst. Thus, only social agents led to robust changes in the participants' decision-making processes, by considering the other's behaviour, in turn disrupting their sense of agency. In line with this, inter-individual differences in perspective taking are related to susceptibility to the bystander effect, with participants higher in perspective taking traits being more strongly affected by the presence of bystanders (Hortensius, Schutter, \& de Gelder, 2016).

In further support of this model, even when explicitly comparing positive and negative action outcomes, we found no indication that the effect of social context on agency was due to a selfserving bias - that is, a strategic displacement of responsibility to other agents for undesirable outcomes. While lower control ratings for worse outcomes might reflect a self-serving bias, our findings show that self-serving bias cannot explain the observed reduction in sense of agency in social contexts. The effect of social context on the sense of agency was not influenced by whether 
participants expected to earn points (gain frame) or avoid losing points (loss frame). Perceived control is an important prerequisite for responsibility: one should reasonably assume more responsibility for a controllable event than for a non-controllable one. Our studies show unequivocally that while participants feel less in control both over undesirable outcomes compared to desirable ones, and also over outcomes achieved in a social vs. non-social context, they do not show a displacement of agency over undesirable outcomes onto their co-player.

Sense of agency is related to a number of perceptual processes (Tsakiris \& Haggard, 2005) and outcome monitoring (Bednark \& Franz, 2014), and is thus presumed to play a crucial role in voluntary action. Previous research has largely focused on the benefits of social contexts to human cognition (Devaine et al., 2014; Vanlangendonck, Takashima, Willems, \& Hagoort, 2018). This has neglected its potentially disruptive effects under some circumstances, as when social context reduces sense of agency and outcome monitoring (Beyer et al., 2017). Our findings have strong implications for common educational practices: reduced sense of agency in social contexts may likely affect feedback-driven learning, making a case for reduced peer influence on individual learning processes. Moreover, future studies should take into account interpersonal variability in the sensitivity to social cues, to better understand the role of mentalizing processes in learning from social feedback, and consequently social development.

\section{Conclusions}

In the presence of other people, mentalizing processes can interfere with non-social aspects of human cognition. In two experiments, we show that the presence of others reduces sense of agency over positive and negative action outcomes, and that this effect is specific to the presence of an intentional, social agent. Our findings suggest that the presence of other people can have 
fundamental effects on how we perceive our own actions and outcomes. This has important implications for our understanding of human behaviour in social environments. Even without an explicit motivation for self-serving displacement of responsibility, the presence of others can affect our subjective sense of agency. An anticipated lack of control might reduce an individual's motivation to take action in a social situation, while reduced outcome monitoring could be linked to reduced learning from action consequences. Thus, further studies should focus on the effects that a reduced sense of agency in social situations might have on subsequent learning and decision-making.

\section{Data availability}

Data is available in de-identified form on Open Science Framework (https://osf.io/2s7kb/). 
Bandura, A. (2002). Selective Moral Disengagement in the Exercise of Moral Agency. Journal of Moral Education, 31(2), 101-119. https://doi.org/10.1080/0305724022014322

Bednark, J. G., \& Franz, E. A. (2014). Agency attribution: event-related potentials and outcome monitoring. Experimental Brain Research, 232(4), 1117-1126. https://doi.org/10.1007/s00221014-3821-4

Beyer, F., Sidarus, N., Bonicalzi, S., \& Haggard, P. (2017). Beyond self-serving bias: diffusion of responsibility reduces sense of agency and outcome monitoring. Social Cognitive and Affective Neuroscience, 12, 138-145.

Beyer, F., Sidarus, N., Fleming, S., \& Haggard, P. (2018). Losing Control in Social Situations: How the Presence of Others Affects Neural Processes Related to Sense of Agency. ENeuro, 5(1), ENEURO.0336-17.2018. https://doi.org/10.1523/ENEURO.0336-17.2018

Devaine, M., Hollard, G., \& Daunizeau, J. (2014). The Social Bayesian Brain: Does Mentalizing Make a Difference When We Learn? PLOS Computational Biology, 10(12), e1003992. https://doi.org/10.1371/journal.pcbi.1003992

Frith, C. D., \& Haggard, P. (2018). Volition and the Brain - Revisiting a Classic Experimental Study. Trends in Neurosciences, 41(7), 405-407. https://doi.org/10.1016/j.tins.2018.04.009

Hare, B. (2011). From Hominoid to Hominid Mind: What Changed and Why? Annual Review of Anthropology, 40(1), 293-309. https://doi.org/10.1146/annurev-anthro-081309-145726

Hon, N., Poh, J.-H., \& Soon, C.-S. (2013). Preoccupied minds feel less control: Sense of agency is modulated by cognitive load. Consciousness and Cognition, 22(2), 556-561.

https://doi.org/10.1016/j.concog.2013.03.004

Hortensius, R., Schutter, D. J. L. G., \& de Gelder, B. (2016). Personal distress and the influence of bystanders on responding to an emergency. Cognitive, Affective \& Behavioral Neuroscience, 16, 672-688. https://doi.org/10.3758/s13415-016-0423-6

Howard, E. E., Edwards, S. G., \& Bayliss, A. P. (2016). Physical and mental effort disrupts the implicit sense of agency. Cognition, 157(Supplement C), 114-125.

https://doi.org/10.1016/j.cognition.2016.08.018

Lejuez, C. W., Read, J. P., Kahler, C. W., Richards, J. B., Ramsey, S. E., Stuart, G. L., ... Brown, R. A. (2002). Evaluation of a behavioral measure of risk taking: The Balloon Analogue Risk Task (BART). Journal of Experimental Psychology: Applied, 8(2), 75.

https://doi.org/10.1037/1076-898X.8.2.75

R Development Core Team. (2008). R: A language and environment for statistical computing. Vienna, Austria: R Foundation for Statistical Computing. Retrieved from http://www.Rproject.org

Schurz, M., Radua, J., Aichhorn, M., Richlan, F., \& Perner, J. (2014). Fractionating theory of mind: A meta-analysis of functional brain imaging studies. Neuroscience \& Biobehavioral Reviews, 42, 9-34. https://doi.org/10.1016/j.neubiorev.2014.01.009 
Sidarus, N., Chambon, V., \& Haggard, P. (2013). Priming of actions increases sense of control over unexpected outcomes. Consciousness and Cognition, 22(4), 1403-1411.

https://doi.org/10.1016/j.concog.2013.09.008

Sidarus, N., \& Haggard, P. (2016). Difficult action decisions reduce the sense of agency: A study using the Eriksen flanker task. Acta Psychologica, 166, 1-11. https://doi.org/10.1016/j.actpsy.2016.03.003

Sidarus, N., Vuorre, M., \& Haggard, P. (2017). How Action Selection Influences the Sense of Agency: An ERP study. NeuroImage. https://doi.org/10.1016/j.neuroimage.2017.02.015

Tom, A. B., Bosker, T. A. S. R. J., \& Bosker, R. J. (1999). Multilevel analysis: an introduction to basic and advanced multilevel modeling. Sage.

Tsakiris $\dagger$, M., \& Haggard, P. (2005). Experimenting with the acting self. Cognitive Neuropsychology, 22(3-4), 387-407. https://doi.org/10.1080/02643290442000158

Vanlangendonck, F., Takashima, A., Willems, R. M., \& Hagoort, P. (2018). Distinguishable memory retrieval networks for collaboratively and non-collaboratively learned information. Neuropsychologia, 111, 123-132. https://doi.org/10.1016/j.neuropsychologia.2017.12.008

Wen, W., Yamashita, A., \& Asama, H. (2016). Divided Attention and Processes Underlying Sense of Agency. Frontiers in Psychology, 7. https://doi.org/10.3389/fpsyg.2016.00035 


\section{SM1 Experiment 1}

\section{SM1a Task performance}

To confirm that participants' task performance did not differ significantly across gain and loss frames, other aspects of participants' behaviour were analysed (means and SDs in table below).

The number of trials in which the participant acted was analysed with a group (gain vs. loss frame) by context (agent absent vs. present) mixed ANOVA. This showed no significant effect of group $\left(\mathrm{F}_{1,41}<.1, \mathrm{p}=.953, \eta_{p}^{2}<.01\right)$, nor a significant interaction between the factors $\left(\mathrm{F}_{1,41}=.1, \mathrm{p}\right.$ $\left.=.817, \eta_{p}^{2}<.01\right)$. A significant main effect of context $\left(\mathrm{F}_{1,41}=221.8, \mathrm{p}<.001, \eta_{p}^{2}=.84\right)$ showed that, across groups, participants acted significantly less often when the alternative agent was present than absent, since the balloon could also be stopped by the co-player.

Analysis of RTs with the same mixed ANOVA revealed no significant main effect of group ( $\mathrm{F}_{1,41}$ $\left.=0.1 ; \mathrm{p}=.759, \eta_{p}^{2}<.01\right)$, nor a significant interaction $\left(\mathrm{F}_{1,41}=1.3 ; \mathrm{p}=.267, \eta_{p}^{2}=.03\right)$. A significant main effect of context $\left(\mathrm{F}_{1,41}=27.4 ; \mathrm{p}<.001, \eta_{p}^{2}=.40\right)$ showed that, across both groups, participants acted significantly later in the agent present than in the agent absent condition. Consistent with our previous findings (Beyer et al 2017), this suggests that participants tended to wait a bit longer to act when an alternative agent was present, since the best outcome was obtained if the co-player acted instead of them. Importantly, participant's behaviour was equally affected by the co-player across gain and loss groups.

Moreover, there was no significant difference across groups in number of trials in which the coplayer acted (in the agent present condition; $\mathrm{t}_{41}=-0.1, \mathrm{p}=.908$ ), nor in participants' final earnings $\left(\mathrm{t}_{41}=0.02, \mathrm{p}=.983\right)$.

\begin{tabular}{|l|l|l|l|l|}
\hline \multirow{2}{*}{ Variable } & \multicolumn{2}{l|}{ Gain Frame } & \multicolumn{2}{l|}{ Loss Frame } \\
\cline { 2 - 5 } & Mean & SD & Mean & SD \\
\hline N participant actions - agent absent & 46.4 & 6.7 & 46.1 & 5.8 \\
\hline N participant actions - agent present & 30.9 & 7.2 & 31.0 & 5.9 \\
\hline Mean RT - agent absent & 6.36 & 0.46 & 6.38 & 0.29 \\
\hline Mean RT - agent present & 6.66 & 0.40 & 6.57 & 0.27 \\
\hline N co-player actions & 15.62 & 3.25 & 15.73 & 2.81 \\
\hline Absolute pence earned & 290 & 24.3 & 290 & 21.6 \\
\hline
\end{tabular}




\section{SM1b Test statistics}

\begin{tabular}{|l|l|l|l|l|l|}
\hline Regressor & $\boldsymbol{\beta}$ & CI & df & t-value & p-value \\
\hline Group & -5.6 & $-15.6 / 4.5$ & 41.0 & -1.1 & .284 \\
\hline Context & 3.0 & $1.2 / 4.8$ & 41.1 & 3.3 & .002 \\
\hline Outcome & 5.3 & $3.9 / 6.8$ & 41.4 & 7.1 & $<.001$ \\
\hline Group*Context & 1.0 & $-2.6 / 4.6$ & 41.1 & .5 & .589 \\
\hline Group*Outcome & -1.6 & $-4.5 / 1.3$ & 41.4 & -1.1 & .294 \\
\hline Context*Outcome & 1.8 & $0.7 / 2.9$ & 39.2 & 3.1 & .003 \\
\hline Group*Context*Outcome & .5 & $-1.8 / 2.7$ & 39.2 & .4 & .693 \\
\hline
\end{tabular}

\section{SM2 Experiment 2}

\section{SM2a Task performance}

We analysed RTs with a group (social and non-social groups) x context (agent absent vs. present) mixed ANOVA (means and SDs in table below). This revealed no significant main effect of group $\left(\mathrm{F}_{1,46}=0.9 ; \mathrm{p}=.358, \eta_{p}^{2}=.02\right)$ or context $\left(\mathrm{F}_{1,46}=1.9 ; \mathrm{p}=.197, \eta_{p}^{2}=.04\right)$, nor a significant interaction $\left(\mathrm{F}_{1,46}=1.2 ; \mathrm{p}=.285, \eta_{p}^{2}=.03\right)$. The absence of any effect on RTs in this experiment suggests that changes in its design and the way the behaviour of the alternative agent was programmed, relative to Exp. 1, may have reduced the variance in RTs. In this design, the number of alternative agent actions was limited at 3 , hence it was less adaptive to wait for the alternative agent, and participants might instead experience more balloon bursts. Nonetheless, as shown in the main article, participants still adapted their behaviour to the presence of the alternative agent by acting less often. Therefore, these task changes did not invalidate the critical effects of context on sense of agency and on decision-making.

\begin{tabular}{|l|l|l|l|l|}
\hline \multirow{2}{*}{ Variable } & \multicolumn{3}{|l|}{ Social Frame } & \multicolumn{2}{l|}{$\begin{array}{l}\text { Non-social } \\
\text { Frame }\end{array}$} \\
\cline { 2 - 5 } & Mean & SD & Mean & SD \\
\hline Mean RT - agent absent & 6.35 & .22 & 6.33 & .21 \\
\hline Mean RT - agent present & 6.33 & .30 & 6.23 & .29 \\
\hline
\end{tabular}


SM2b Test statistics full model

\begin{tabular}{|l|l|l|l|l|l|}
\hline Regressor & $\boldsymbol{\beta}$ & CI & df & t-value & p-value \\
\hline Group & -6.2 & $-17.5 / 5.1$ & 46.1 & -1.1 & .286 \\
\hline Context & 1.6 & $-.6 / 3.8$ & 44.2 & 1.5 & .154 \\
\hline Outcome & 5.13 & $3.4 / 6.8$ & 46.4 & 5.9 & $<.001$ \\
\hline Group*Context & 4.0 & $-.4 / 8.3$ & 44.2 & 1.8 & .079 \\
\hline Group*Outcome & 1.2 & $-2.2 / 4.6$ & 46.4 & 0.7 & .489 \\
\hline Context*Outcome & .8 & $-.8 / 2.3$ & 84.4 & 1.0 & .342 \\
\hline Group*Context*Outcome & 4.3 & $1.2 / 7.4$ & 84.4 & 2.7 & .008 \\
\hline
\end{tabular}

SM2c Test statistics social group

\begin{tabular}{|l|l|l|l|l|l|}
\hline Regressor & $\boldsymbol{\beta}$ & CI & df & t-value & p-value \\
\hline Context & 3.6 & $-.4 / 7.6$ & 22.5 & 1.8 & .091 \\
\hline Outcome & 5.75 & $3.4 / 8.1$ & 23.4 & 4.81 & $<.001$ \\
\hline Context*Outcome & 2.85 & $.2 / 5.46$ & 40.6 & 2.1 & .04 \\
\hline
\end{tabular}

\section{SM2d Test statistics non-social group}

\begin{tabular}{|l|l|l|l|l|l|}
\hline Regressor & $\boldsymbol{\beta}$ & CI & df & t-value & p-value \\
\hline Context & -.4 & $-2.4 / 1.5$ & 32.2 & -.4 & .671 \\
\hline Outcome & 4.6 & $2.2 / 7.0$ & 23.1 & 3.7 & .001 \\
\hline Context*Outcome & -1.5 & $-3.2 / 0.3$ & 33.0 & -1.6 & .115 \\
\hline
\end{tabular}

